\title{
Mid-Infrared Supercontinuum Generation in Germanium Waveguides
}

\author{
Alberto Della Torre, ${ }^{1}$ Milan Sinobad, ${ }^{1}$ Rémi Armand, ${ }^{1}$ Barry Luther-Davies, ${ }^{2}$ Pan $M a,{ }^{2}$ Stephen Madden, ${ }^{2}$ \\ David J. Moss, ${ }^{3}$ Arnan Mitchell, ${ }^{4}$ Jean-Michel Hartmann, ${ }^{5}$ Vincent Reboud, ${ }^{5}$ Jean-Marc Fedeli, ${ }^{5}$ \\ Christelle Monat, ${ }^{1}$ and Christian Grillet ${ }^{1}$ \\ ${ }^{1}$ Université de Lyon, Institut des Nanotechnologies de Lyon (INL), 69131 Ecully, France \\ ${ }^{2}$ Laser Physics Centre, Australian National University, Canberra, ACT 0100, Australia \\ ${ }^{3}$ Optical Sciences Centre, Swinburne University of Technology, Hawthorn, VIC 3122, Australia \\ ${ }^{4}$ School of Engineering, RMIT University, Melbourne, VIC 3001, Australia \\ ${ }^{5}$ Université Grenoble Alpes, CEA-Leti, 38054 Grenoble Cedex 9, France
}

On-chip mid-infrared (mid-IR, from 3 to $15 \mu \mathrm{m}$ wavelength) supercontinuum (SC) generation is of great interest for integrated sensing devices, since it enables parallel detection of multiples species [1]. The generation of midIR SC in group IV platforms is particularly significant, since it would allow low-cost, large-scale production and electro-optic integration. Mid-IR SC generation has been demonstrated in several group-IV platforms [1-4]. Recently, the wide transparency window up to $15 \mu \mathrm{m}$ and theoretical predictions of attractive nonlinear properties [5] led researchers to explore germanium as a promising material for mid-infrared photonics. However, experimental SC generation in pure germanium waveguides remained elusive so far. Our group reported SC generation up to $8.5 \mu \mathrm{m}$ in silicon-germanium on silicon waveguides [3]. We have also demonstrated the possibility, with this platform, of precisely controlling the SC coherence properties [6-8]. The great potential of germanium-based platforms for on-chip mid-IR SC generation was recently confirmed, with the demonstration of a SC extending from 3 up to $13 \mu \mathrm{m}$ in a germanium-rich graded index silicon-germanium on silicon waveguide [9]. To achieve such a broad spectrum, however, the waveguide was pumped at $7.5 \mu \mathrm{m}$, whereas short wavelength pumps are preferable in fully integrated broadband sources.

Here, we report the first experimental demonstration of SC generation in a pure germanium waveguide, and we investigate the origins of the bandwidth limitations when pumping at shorter wavelengths [10]. To those ends, we pumped a $4.46 \mu \mathrm{m}$ wide, $2.57 \mu \mathrm{m}$ thick germanium on silicon air-clad waveguide (fig. 1a, inset) with $200 \mathrm{fs}$ pulses at $4.6 \mu \mathrm{m}$ (close to the zero dispersion wavelength, see fig. 1a) and generate a SC extending from 3.53 to $5.83 \mu \mathrm{m}$ (fig. 1b, top), with milliwatt-level on-chip power. With the help of numerical simulations, we attribute the long wavelength extension limit of the SC to absorption from free-carriers $\left(\alpha_{\mathrm{FC}}\right)$, generated by three-photon absorption. Indeed, simulations considering constant $\alpha_{\mathrm{FC}}$ predict SC generation up to $7 \mu \mathrm{m}$ (fig. $1 \mathrm{~b}$, bottom, green curve), whereas simulations including the wavelength dependence of $\alpha_{\mathrm{FC}}$ (fig. 1b, bottom, blue curve) correctly reproduce the experimental SC. Owing to the transparency of the atmosphere between 3 and $5 \mu \mathrm{m}$ and to the strong absorption of hazardous and greenhouse gases such as $\mathrm{CO}(\sim 4.5 \mu \mathrm{m}), \mathrm{CO}_{2}(4.2,4,3 \mu \mathrm{m})$ and $\mathrm{CH}_{4}(3.45 \mu \mathrm{m})$, our source has potential applications in free-space communications and environmental monitoring.
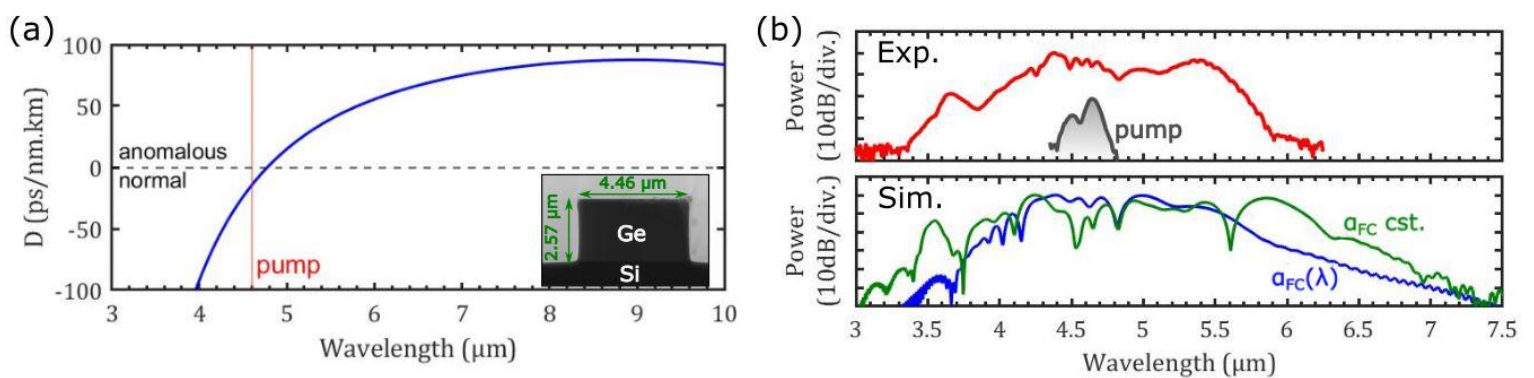

Fig. 1 (a) Dispersion curve of the germanium-on silicon waveguide (SEM image shown in the inset). (b) Experimental (top) and simulated (bottom, green curve considering constant free-carrier absorption, blue curve considering its wavelength dependence) supercontinuum.

Funding. We acknowledge the support of the International Associated Laboratory in Photonics between France and Australia (LIA ALPhFA), the Agence Nationale de la Recherche (MIRSICOMB, ANR-17-CE24-0028) and the European Research Council (ERC) under the European Union's Horizon 2020 program (GRAPHICS 648546).

\section{References}

[1] E. Tagkoudi et al., Opt. Lett. 45, 2195-2198 (2020).

[2] N. Singh et al., Optica 2, 797-802 (2015).

[3] M. Sinobad et al., Optica 5, 360-366 (2018).

[4] N. Nader et al., Optica 6, 1269-1276 (2019).

[5] N. K. Hon, R. Soref, and B. Jalali, J. Appl. Phys. 110, 011301 (2011).

[6] M. Sinobad et al., J. Opt. Soc. Am. B 36, A98-A104 (2019).

[7] M. Sinobad et al., IEEE J. Sel. Top. Quantum Electron. 26, 1-8 (2019).

[8] M. Sinobad et al., Opt. Lett. 45, 5008-5011 (2020).

[9] M. Montesinos-Ballester et al., ACS Photonics 26, 3423-3429 (2020).

[10] A. Della Torre et al., APL Photonics 6, 016102 (2021). 